\title{
Sensitivity analysis of reactive ecological dynamics
}

\author{
Ariane Verdy \\ Department of Earth, Atmospheric and Planetary Sciences, \\ Massachusetts Institute of Technology, Cambridge, MA 02139 (averdy@mit.edu)
}

\author{
Hal Caswell
}

Biology Department MS-34, Woods Hole Oceanographic Institution, Woods Hole, MA 02543 (hcaswell@whoi.edu)

Manuscript published in the Bulletin of Mathematical Biology

August 2008

DOI 10.1007/s11538-008-9312-7 


\begin{abstract}
Ecological systems with asymptotically stable equilibria may exhibit significant transient dynamics following perturbations. In some cases, these transient dynamics include the possibility of excursions away from the equilibrium before the eventual return; systems that exhibit such amplification of perturbations are called reactive. Reactivity is a common property of ecological systems, and the amplification can be large and longlasting. The transient response of a reactive ecosystem depends on the parameters of the underlying model. To investigate this dependence, we develop sensitivity analyses for indices of transient dynamics (reactivity, the amplification envelope, and the optimal perturbation) in both continuous- and discrete-time models written in matrix form. The sensitivity calculations require expressions, some of them new, for the derivatives of equilibria, eigenvalues, singular values, and singular vectors, obtained using matrix calculus. Sensitivity analysis provides a quantitative framework for investigating the mechanisms leading to transient growth. We apply the methodology to a predator-prey model and a size-structured food web model. The results suggest predator-driven and prey-driven mechanisms for transient amplification resulting from multispecies interactions.
\end{abstract}

Keywords: Ecological models, transient dynamics, reactivity, sensitivity analysis, consumer-resource interactions, matrix population models. 


\section{Introduction}

Sufficiently small perturbations of an asymptotically stable equilibrium will eventually decay. The asymptotic rate of return to equilibrium has long been used as a measure of ecological stability (e.g., May, 1973; Pimm, 1984; Ives and Carpenter, 2007). However, the long-term return to the equilibrium does not determine the transient response to the perturbation, which may carry the trajectory farther away from the equilibrium before its eventual return. Equilibria with this property are called "reactive" (Neubert and Caswell, 1997). It is now known that reactivity is a common property of predator-prey models, food web models, ecosystem compartment models, and stage-classified matrix population models (Neubert and Caswell, 1997; Caswell, 2001; Chen and Cohen, 2001; Neubert et al, 2004; Marvier et al, 2004; Caswell and Neubert, 2005). Reactivity has been shown to be a necessary condition for pattern formation via Turing instability (Neubert et al, 2002).

The transient amplification of perturbations is important because ecological systems may not complete their response to a perturbation before the next one occurs. Instead, they are buffeted by a more-or-less continual series of perturbations and the appearance of transient responses in our observations of nature may be the norm rather than the exception (Hastings, 2004). Managers charged with ecosystem restoration, for example, are likely to be interested in both the short-term and long-term effects of their manipulations (cf. Caswell, 2007), particularly if the short-term effects can be large.

To understand transient dynamics, it is useful to know how their properties respond to the parameters in the underlying model. To this end, we present here the sensitivity analysis of several properties of reactive transient dynamics. The paper begins with a brief introduction of the indices used to describe transient growth, followed by a description of the sensitivity problem. Section 2 deals with the calculation of the sensitivity of equilibrium solutions and of linearized dynamics; these are used to calculate sensitivities of indices of transient dynamics in Section 3. The method is applied to two ecological problems in Section 4 and briefly discussed in Section 5 . 


\subsection{Characterizing transient dynamics}

The stability and asymptotic properties of an equilibrium are determined by the dominant and subdominant eigenvalues of the linearization of the model at that equilibrium. Simple analysis of these eigenvalues does not, however, capture the transient behavior of systems whose time evolution is described by non-normal matrices or operators (Trefethen and Embree, 2005). Mathematical developments in areas such as fluid dynamics (e.g., Farrell and Ioannou, 1996; Trefethen et al, 1993) and numerical analysis (e.g., Trefethen, 1992) have led to the formulation of indices that characterize the transient response of nonlinear ecological systems (Neubert and Caswell, 1997).

We focus on three such indices of transient dynamics. The reactivity of an asymptotically stable equilibrium is the maximum, over all perturbations, of the rate at which the trajectory departs from the equilibrium in the linearized system (or equivalently, for sufficiently small perturbations). It measures the maximum instantaneous amplification of perturbations of that equilibrium. At any time following a perturbation, there is a maximum (again, over all perturbations) possible deviation from the equilibrium. This maximum is the amplification envelope (Neubert and Caswell, 1997). It provides an upper bound on the extent of transient amplification as a function of time. Transient amplification depends on the direction of the initial perturbation. The perturbation that produces the maximum amplification at a specified time is the optimal perturbation, also called the optimal excitation (Farrell and Ioannou, 1996). The reactivity, amplification envelope, and optimal perturbation are important descriptions of the behavior of ecosystems subject to random perturbations. They provide information about the timing and magnitude of the growth of perturbations, the potential for transient amplification, and the perturbations to which the system is most sensitive.

We consider both continuous and discrete models, written in matrix form:

$$
\begin{aligned}
\frac{d \mathbf{x}}{d t} & =\mathbf{A}[\boldsymbol{\theta}, \mathbf{x}] \mathbf{x} & & \text { continuous } \\
\mathbf{x}(t+1) & =\mathbf{A}[\boldsymbol{\theta}, \mathbf{x}] \mathbf{x}(t) & & \text { discrete }
\end{aligned}
$$


where $\mathbf{x}$ is a vector $(s \times 1)$ of state variables and the matrix $\mathbf{A}$ contains per-capita vital rates. The vital rates depend on the current system state $\mathbf{x}$ and on a vector $\boldsymbol{\theta}$ of parameters. Many ecological systems, including structured populations, interacting species, food webs, compartment models, and epidemic models can be written in this form. When it seems unlikely to cause confusion, we will suppress the explicit dependence of $\mathbf{A}$ on $\mathbf{x}$ and $\boldsymbol{\theta}$.

Let $\hat{\mathbf{x}}$ be an equilibrium. The linearization around $\hat{\mathbf{x}}$ is given by the Jacobian matrix $\mathbf{M}=\mathbf{M}[\boldsymbol{\theta}, \hat{\mathbf{x}}]$. Deviations from $\hat{\mathbf{x}}$, defined as $\mathbf{z}(t)=\mathbf{x}(t)-\hat{\mathbf{x}}$, follow

$$
\begin{aligned}
\frac{d \mathbf{z}}{d t} & =\mathbf{M}[\boldsymbol{\theta}, \hat{\mathbf{x}}] \mathbf{z} & & \text { continuous } \\
\mathbf{z}(t+1) & =\mathbf{M}[\boldsymbol{\theta}, \hat{\mathbf{x}}] \mathbf{z}(t) & & \text { discrete }
\end{aligned}
$$

We assume that $\hat{\mathbf{x}}$ is asymptotically stable, so that the dominant eigenvalue of $\mathbf{M}$ has negative real part (in continuous time) or is less than 1 in magnitude (in discrete time).

The transient dynamics of the perturbed system are described by the evolution of the magnitude of $\mathbf{z}$, as measured by the Euclidian norm $\|\mathbf{z}\|=\sqrt{\mathbf{z}^{\top} \mathbf{z}}$. We consider the transient response following a perturbation $\mathbf{z}_{0}$ at $t=0$. The reactivity is the maximum, over all perturbations, of the growth rate of $\|\mathbf{z}\|$, as $t \rightarrow 0$.

In continuous time, the reactivity is

$$
\begin{aligned}
\nu_{0} & =\left.\max _{\left\|\mathbf{z}_{0}\right\| \neq 0} \frac{1}{\|\mathbf{z}\|} \frac{d\|\mathbf{z}\|}{d t}\right|_{t=0} \\
& =\max _{\left\|\mathbf{z}_{0}\right\| \neq 0} \frac{\mathbf{z}_{0}^{\top}\left(\mathbf{M}+\mathbf{M}^{\top}\right) \mathbf{z}_{0}}{2 \mathbf{z}_{0}^{\top} \mathbf{z}_{0}} \\
& =\lambda_{1}(\mathbf{H}(\mathbf{M}))
\end{aligned}
$$

where $\mathbf{H}(\mathbf{M})=\left(\mathbf{M}+\mathbf{M}^{\top}\right) / 2$ is the Hermitian part of $\mathbf{M}$ and $\lambda_{1}$ denotes the eigenvalue with largest real part (Neubert and Caswell, 1997).

In discrete time, reactivity is defined as the average instantaneous rate of growth, from $t=0$ to $t=1$, following the perturbation $\mathbf{z}_{0}$ :

$$
\nu_{0}=\log \left(\max _{\|\mathbf{z}\| \neq 0} \frac{\left\|\mathbf{M} \mathbf{z}_{0}\right\|}{\left\|\mathbf{z}_{0}\right\|}\right)
$$




$$
\begin{aligned}
& =\log \|\mathbf{M}\| \mid \\
& =\log \left(\sigma_{1}(\mathbf{M})\right)
\end{aligned}
$$

where $\log (\cdot)$ is the natural $\operatorname{logarithm}$, and the matrix norm $\||\|| \mid$ induced by the Euclidian norm is the largest singular value, denoted by $\sigma_{1}$. In either continuous or discrete time, if $\nu_{0}>0$ there exists a perturbation that produces a trajectory departing from $\hat{\mathbf{x}}$ at the rate $\nu_{0}$.

To obtain the amplification envelope, we solve (3) and (4) as

$$
\mathbf{z}(t)=\mathbf{\Phi}(t) \mathbf{z}_{0}
$$

where $\boldsymbol{\Phi}(t)$ is the fundamental matrix ${ }^{1}$ (Coddington and Levinson, 1955, p. 69), given by

$$
\boldsymbol{\Phi}(t)= \begin{cases}e^{\mathbf{M} t} & \text { continuous } \\ \mathbf{M}^{t} & \text { discrete }\end{cases}
$$

and the matrix exponential is defined as $e^{\mathbf{M} t}=\sum_{i=0}^{\infty} \frac{(\mathbf{M} t)^{i}}{i !}$.

The amplification envelope at time $t$ is the maximum, over all initial perturbations, of the growth of $\mathbf{z}(t)$,

$$
\begin{aligned}
\rho(t) & =\max _{\left\|\mathbf{z}_{0}\right\| \neq 0} \frac{\|\mathbf{z}(t)\|}{\left\|\mathbf{z}_{0}\right\|} \\
& =\|\mathbf{\Phi}(t)\| \\
& =\sigma_{1}(\mathbf{\Phi}(t))
\end{aligned}
$$

The optimal perturbation, normalized to length 1 , is given by the right singular vector $\mathbf{v}(t)$ corresponding to the singular value $\sigma_{1}(\boldsymbol{\Phi}(t))$.

\footnotetext{
${ }^{1}$ Also called the propagator (Farrell and Ioannou, 1996), or matricant (e.g., Gantmacher, 1959, p. 125).
} 


\subsection{The sensitivity problem}

We can define the sensitivity problem for these three indices of transient dynamics. Let $r$ denote one of these indices; $r$ is a function of the linearization $\mathbf{M}$, which in turn depends on the parameters and the equilibrium. Our goal is to obtain the sensitivities

$$
\frac{d r}{d \boldsymbol{\theta}^{\top}}
$$

and the elasticities

$$
\frac{1}{r} \frac{d r}{d \boldsymbol{\theta}^{\top}} \operatorname{diag}(\boldsymbol{\theta})
$$

in a way that accounts for both the direct effects of $\boldsymbol{\theta}$ on $\mathbf{M}$ and the indirect effects of $\boldsymbol{\theta}$ on M through $\hat{\mathbf{x}}$.

Making use of the chain rule, we write

$$
\frac{d r}{d \boldsymbol{\theta}^{\top}}=\frac{d r}{d \operatorname{vec}^{\top} \mathbf{M}} \frac{d \operatorname{vec} \mathbf{M}}{d \boldsymbol{\theta}^{\top}}
$$

which, because $\mathbf{M}$ depends on both $\hat{\mathbf{x}}$ and $\boldsymbol{\theta}$, expands to

$$
\frac{d r}{d \boldsymbol{\theta}^{\top}}=\left(\frac{d r}{d \operatorname{vec}^{\top} \mathbf{M}}\right)\left(\frac{\partial \operatorname{vec} \mathbf{M}}{\partial \boldsymbol{\theta}^{\top}}+\frac{\partial \operatorname{vec} \mathbf{M}}{\partial \hat{\mathbf{x}}^{\top}} \frac{d \hat{\mathbf{x}}}{d \boldsymbol{\theta}^{\top}}\right)
$$

The sensitivity of $r$ in (19) requires four pieces: the linearization $\mathbf{M}$ at the equilibrium, the sensitivity of the equilibrium $\hat{\mathbf{x}}$ to the parameters, the sensitivity of the linearization to the parameters, and the sensitivity of $r$ to the linearization.

These derivatives are written using the matrix calculus conventions of Magnus and Neudecker $(1985,1988)$ [see Nel (1980) for a review, and Caswell (2007) for an ecological introduction]. In this approach, the derivative of a $n \times 1$ vector $\mathbf{y}$ with respect to a $m \times 1$ vector $\mathbf{x}$ is the $n \times m$ Jacobian matrix

$$
\frac{d \mathbf{y}}{d \mathbf{x}^{\top}}=\left(\frac{d y_{i}}{d x_{j}}\right)
$$

Derivatives of, or with respect to, matrices are converted to vector derivatives using the 
vec operator, which stacks the columns of a matrix one above the other (the first column is on top and the last one on the bottom). Thus the derivative of the $m \times n$ matrix $\mathbf{Y}$ with respect to the $p \times q$ matrix $\mathbf{X}$ is the $m n \times p q$ matrix

$$
\frac{d \operatorname{vec} \mathbf{Y}}{d \operatorname{vec}^{\top} \mathbf{X}}
$$

where $\operatorname{vec}^{\top} \mathbf{X}=(\operatorname{vec} \mathbf{X})^{\top}$. We make frequent use of the result (Roth, 1934) that

$$
\operatorname{vec}(\mathbf{A B C})=\left(\mathbf{C}^{\top} \otimes \mathbf{A}\right) \operatorname{vec} \mathbf{B}
$$

The Kronecker product of two matrices is defined as

$$
\mathbf{A} \otimes \mathbf{B}=\left(\begin{array}{ccc}
a_{11} \mathbf{B} & a_{12} \mathbf{B} & \ldots \\
a_{21} \mathbf{B} & a_{22} \mathbf{B} & \ldots \\
\vdots & \vdots & \ddots
\end{array}\right)
$$

\section{Equilibria, linearizations, and their sensitivities}

In this section, we present the analysis of the linearization and the equilibrium. In Section 3 we will combine these to obtain the sensitivity of reactivity, the amplification envelope, and the optimal perturbation.

\subsection{The linearization}

The matrix of the linearization at an equilibrium $\hat{\mathbf{x}}$ is

$$
\mathbf{M}= \begin{cases}\left.\frac{\partial \dot{\mathbf{x}}}{\partial \mathbf{x}^{\top}}\right|_{\hat{\mathbf{x}}} & \text { continuous } \\ \left.\frac{\partial \mathbf{x}(t+1)}{\partial \mathbf{x}^{\top}(t)}\right|_{\hat{\mathbf{x}}} & \text { discrete }\end{cases}
$$

where $\dot{\mathbf{x}}=d \mathbf{x} / d t$ is given in (1) and $\mathbf{x}(t+1)$ is given in (2). Because we have written the models in matrix form, the expression for $\mathbf{M}$ is the same for both models; here is the derivation for the continuous case. 
Differentiating $\dot{\mathbf{x}}$ in (1) gives

$$
d \dot{\mathbf{x}}=(d \mathbf{A}) \mathbf{x}+\mathbf{A}(d \mathbf{x})
$$

Applying the vec operator to both sides gives

$$
d \dot{\mathbf{x}}=\left(\mathbf{x}^{\top} \otimes \mathbf{I}_{s}\right) d \operatorname{vec} \mathbf{A}+\mathbf{A} d \mathbf{x}
$$

from which

$$
\mathbf{M}=\left(\mathbf{x}^{\top} \otimes \mathbf{I}_{s}\right) \frac{d \mathrm{vec} \mathbf{A}}{d \mathbf{x}^{\top}}+\mathbf{A}
$$

where $\mathbf{I}_{s}$ is an identity matrix of order $s$. The linearization at the equilibrium is obtained by evaluating $\mathbf{M}$ at $\mathbf{x}=\hat{\mathbf{x}}$ :

$$
\mathbf{M}[\boldsymbol{\theta}, \hat{\mathbf{x}}]=\left(\hat{\mathbf{x}}^{\top} \otimes \mathbf{I}_{s}\right) \frac{\partial \mathrm{vec} \mathbf{A}[\boldsymbol{\theta}, \hat{\mathbf{x}}]}{\partial \mathbf{x}^{\top}}+\mathbf{A}[\boldsymbol{\theta}, \hat{\mathbf{x}}]
$$

\subsection{Sensitivity of equilibria}

The equilibrium $\hat{\mathbf{x}}$ depends on the parameters through the entries in $\mathbf{A}[\boldsymbol{\theta}, \mathbf{x}]$. Its sensitivity is obtained by differentiating the equations defining the equilibrium (see Appendix A for the continuous case, and Caswell (2008) for the discrete case). The sensitivity in the continuous case is

$$
\frac{d \hat{\mathbf{x}}}{d \boldsymbol{\theta}^{\top}}=\left\{-\mathbf{A}-\left(\hat{\mathbf{x}}^{\top} \otimes \mathbf{I}_{s}\right) \frac{\partial \operatorname{vec} \mathbf{A}}{\partial \mathbf{x}^{\top}}\right\}^{-1}\left(\hat{\mathbf{x}}^{\top} \otimes \mathbf{I}_{s}\right) \frac{\partial \operatorname{vec} \mathbf{A}}{\partial \boldsymbol{\theta}^{\top}}
$$

The sensitivity in the discrete case is

$$
\frac{d \hat{\mathbf{x}}}{d \boldsymbol{\theta}^{\top}}=\left\{\mathbf{I}_{s}-\mathbf{A}-\left(\hat{\mathbf{x}}^{\top} \otimes \mathbf{I}_{s}\right) \frac{\partial \operatorname{vec} \mathbf{A}}{\partial \mathbf{x}^{\top}}\right\}^{-1}\left(\hat{\mathbf{x}}^{\top} \otimes \mathbf{I}_{s}\right) \frac{\partial \mathrm{vec} \mathbf{A}}{\partial \boldsymbol{\theta}^{\top}}
$$

In both expressions, the matrix $\mathbf{A}$ and all its derivatives are evaluated at $\hat{\mathbf{x}}$. 


\subsection{Sensitivity of the linearization}

To obtain the sensitivity of the linearization, we differentiate Eq. (28) for $\mathbf{M}[\boldsymbol{\theta}, \hat{\mathbf{x}}]$ :

$$
d \mathbf{M}=\left\{d\left(\hat{\mathbf{x}}^{\top} \otimes \mathbf{I}_{s}\right)\right\} \frac{\partial \mathrm{vec} \mathbf{A}}{\partial \mathbf{x}^{\top}}+\left(\hat{\mathbf{x}}^{\top} \otimes \mathbf{I}_{s}\right) d\left(\frac{\partial \mathrm{vec} \mathbf{A}}{\partial \mathbf{x}^{\top}}\right)+d \mathbf{A}
$$

Applying the vec operator to both sides gives

$$
\begin{aligned}
d \operatorname{vec} \mathbf{M}= & \left\{\left(\frac{\partial \operatorname{vec} \mathbf{A}}{\partial \mathbf{x}^{\top}}\right)^{\top} \otimes \mathbf{I}_{s}\right\} d \operatorname{vec}\left(\hat{\mathbf{x}}^{\top} \otimes \mathbf{I}_{s}\right) \\
& +\left\{\mathbf{I}_{s} \otimes\left(\hat{\mathbf{x}}^{\top} \otimes \mathbf{I}_{s}\right)\right\} d \operatorname{vec}\left(\frac{\partial \operatorname{vec} \mathbf{A}}{\partial \mathbf{x}^{\top}}\right)+d \operatorname{vec} \mathbf{A}
\end{aligned}
$$

Theorem 11 of Magnus and Neudecker (1985), for the differential of a Kronecker product, implies that

$$
d\left[\operatorname{vec}\left(\hat{\mathbf{x}}^{\top} \otimes \mathbf{I}_{s}\right)\right]=\left(\mathbf{I}_{s} \otimes \operatorname{vec} \mathbf{I}_{s}\right) d \hat{\mathbf{x}}
$$

To differentiate $\partial \mathrm{vec} \mathbf{A} / \partial \mathbf{x}^{\top}$, define

$$
\mathbf{B}[\boldsymbol{\theta}, \hat{\mathbf{x}}] \equiv \frac{d \operatorname{vec} \mathbf{A}}{d \mathbf{x}^{\top}}
$$

Then

$$
d \operatorname{vec} \mathbf{B}=\frac{\partial \operatorname{vec} \mathbf{B}}{\partial \boldsymbol{\theta}^{\top}} d \boldsymbol{\theta}+\frac{\partial \operatorname{vec} \mathbf{B}}{\partial \mathbf{x}^{\top}} \frac{d \hat{\mathbf{x}}}{d \boldsymbol{\theta}^{\top}} d \boldsymbol{\theta}
$$

Similarly,

$$
d \operatorname{vec} \mathbf{A}=\frac{\partial \operatorname{vec} \mathbf{A}}{\partial \boldsymbol{\theta}^{\top}} d \boldsymbol{\theta}+\frac{\partial \operatorname{vec} \mathbf{A}}{\partial \mathbf{x}^{\top}} \frac{d \hat{\mathbf{x}}}{d \boldsymbol{\theta}^{\top}} d \boldsymbol{\theta}
$$

Substituting (33), (35), and (36) into (32) and collecting terms gives

$$
\begin{aligned}
\frac{d \operatorname{vec} \mathbf{M}}{d \boldsymbol{\theta}^{\top}}= & \left\{\left(\frac{\partial \operatorname{vec} \mathbf{A}}{\partial \mathbf{x}^{\top}}\right)^{\top} \otimes \mathbf{I}_{s}\right\}\left(\mathbf{I}_{s} \otimes \operatorname{vec} \mathbf{I}_{s}\right) \frac{d \hat{\mathbf{x}}}{d \boldsymbol{\theta}^{\top}} \\
& +\left\{\mathbf{I}_{s} \otimes\left(\hat{\mathbf{x}}^{\top} \otimes \mathbf{I}_{s}\right)\right\}\left(\frac{\partial \operatorname{vec} \mathbf{B}}{\partial \boldsymbol{\theta}^{\top}}+\frac{\partial \operatorname{vec} \mathbf{B}}{\partial \mathbf{x}^{\top}} \frac{d \hat{\mathbf{x}}}{d \boldsymbol{\theta}^{\top}}\right)+\frac{\partial \operatorname{vec} \mathbf{A}}{\partial \boldsymbol{\theta}^{\top}}+\frac{\partial \operatorname{vec} \mathbf{A}}{\partial \mathbf{x}^{\top}} \frac{d \hat{\mathbf{x}}}{d \boldsymbol{\theta}^{\top}}
\end{aligned}
$$


where all matrices and derivatives are evaluated at $\hat{\mathbf{x}}$, and where $d \hat{\mathbf{x}} / d \boldsymbol{\theta}^{\top}$ is given by (29) for continuous models and (30) for discrete models.

\section{Sensitivity of transient indices}

We turn now to the sensitivity of reactivity, the amplification envelope, and the optimal perturbation. To do so, we must find the sensitivity of each index to the linearization $\mathbf{M}[\boldsymbol{\theta}, \hat{\mathbf{x}}]$ and then, following (19), combine this with the sensitivity of $\mathbf{M}$ as given in (37).

\subsection{Sensitivity of reactivity}

In continuous systems, the reactivity $\nu_{0}$ is the dominant eigenvalue of the matrix $\mathbf{H}(\mathbf{M})=$ $\left(\mathbf{M}+\mathbf{M}^{\top}\right) / 2$. Appendix B.1 shows that differentiating $\nu_{0}$ with respect to $\mathbf{M}$ gives

$$
\frac{d \nu_{0}}{d \operatorname{vec}^{\top} \mathbf{M}}=\left(\mathbf{w}^{\top} \otimes \mathbf{w}^{\top}\right)
$$

where $\mathbf{w}$ is the eigenvector of $\mathbf{H}(\mathbf{M})$ associated with the eigenvalue $\nu_{0}$ (Magnus and Neudecker, 1988). This result is given in different notation in Neubert and Caswell (1997).

In discrete systems, the reactivity is the logarithm of the dominant singular value $\sigma_{1}$ of M. Its derivative is (cf. Stewart, 1991; Caswell and Neubert, 2005)

$$
\frac{d \nu_{0}}{d \operatorname{vec}^{\top} \mathbf{M}}=\frac{1}{\|\mathbf{M}\|}\left(\mathbf{v}^{\top} \otimes \mathbf{u}^{\top}\right)
$$

where $\mathbf{u}$ is the left singular vector and $\mathbf{v}$ is the right singular vector of $\mathbf{M}$ corresponding to $\sigma_{1}$. Thus the sensitivity of reactivity is

$$
\frac{d \nu_{0}}{d \boldsymbol{\theta}^{\top}}= \begin{cases}\left(\mathbf{w}^{\top} \otimes \mathbf{w}^{\top}\right) \frac{d \operatorname{vec} \mathbf{M}}{d \boldsymbol{\theta}^{\top}} & \text { continuous } \\ \frac{1}{\|\mathbf{M}\| \mid}\left(\mathbf{v}^{\top} \otimes \mathbf{u}^{\top}\right) \frac{d \operatorname{vec} \mathbf{M}}{d \boldsymbol{\theta}^{\top}} & \text { discrete }\end{cases}
$$

where $d \operatorname{vec} \mathbf{M} / d \boldsymbol{\theta}^{\top}$ is given by (37). 


\subsection{Sensitivity of the amplification envelope}

The amplification envelope $\rho(t)$ is the largest singular value of $\boldsymbol{\Phi}(t)$. Its derivative is

$$
\frac{d \rho(t)}{d \operatorname{vec}^{\top} \mathbf{M}}=\frac{d \rho(t)}{d \operatorname{vec}^{\top} \boldsymbol{\Phi}(t)} \frac{d \operatorname{vec} \boldsymbol{\Phi}(t)}{d \operatorname{vec}^{\top} \mathbf{M}}
$$

The derivative of the singular value is

$$
\frac{d \rho(t)}{d \operatorname{vec}^{\top} \boldsymbol{\Phi}(t)}=\left(\mathbf{v}^{\top}(t) \otimes \mathbf{u}^{\top}(t)\right)
$$

where $\mathbf{u}(t)$ and $\mathbf{v}(t)$ are the left and right singular vectors of $\boldsymbol{\Phi}(t)$ corresponding to $\sigma_{1}$.

In continuous time, the derivative of the fundamental matrix is

$$
\begin{aligned}
\frac{d \operatorname{vec} \boldsymbol{\Phi}(t)}{d \operatorname{vec}^{\top} \mathbf{M}} & =\frac{d \operatorname{vec} e^{\mathbf{M} t}}{d \operatorname{vec}^{\top} \mathbf{M}} \\
& =\sum_{i=0}^{\infty} \frac{t^{i}}{i !} \sum_{j=1}^{i}\left(\mathbf{M}^{\top}\right)^{i-j} \otimes \mathbf{M}^{j-1}
\end{aligned}
$$

The equivalent in discrete time is

$$
\begin{aligned}
\frac{d \operatorname{vec} \boldsymbol{\Phi}(t)}{d \operatorname{vec}^{\top} \mathbf{M}} & =\frac{d \operatorname{vec} \mathbf{M}^{t}}{d \operatorname{vec}^{\top} \mathbf{M}} \\
& =\sum_{j=1}^{t}\left(\mathbf{M}^{\top}\right)^{t-j} \otimes \mathbf{M}^{j-1}
\end{aligned}
$$

Thus the sensitivity of the amplification envelope is

$$
\frac{d \rho(t)}{d \boldsymbol{\theta}^{\top}}= \begin{cases}\left(\mathbf{v}^{\top}(t) \otimes \mathbf{u}^{\top}(t)\right)\left(\sum_{i=0}^{\infty} \frac{t^{i}}{i !} \sum_{j=1}^{i}\left(\mathbf{M}^{\top}\right)^{i-j} \otimes \mathbf{M}^{j-1}\right) \frac{d \mathrm{vec} \mathbf{M}}{d \boldsymbol{\theta}^{\top}} & \text { continuous } \\ \left(\mathbf{v}^{\top}(t) \otimes \mathbf{u}^{\top}(t)\right)\left(\sum_{j=1}^{t}\left(\mathbf{M}^{\top}\right)^{t-j} \otimes \mathbf{M}^{j-1}\right) \frac{d \mathrm{vec} \mathbf{M}}{d \boldsymbol{\theta}^{\top}} & \text { discrete }\end{cases}
$$

where $d \operatorname{vec} \mathbf{M} / d \boldsymbol{\theta}^{\top}$ is given by (37). 


\subsection{Sensitivity of the optimal perturbation}

The optimal perturbation is given by $\mathbf{v}(t)$, the leading right singular vector of $\boldsymbol{\Phi}(t)$. To calculate the sensitivity of $\mathbf{v}(t)$ we require the sensitivity of the singular vector to the matrix $\mathbf{\Phi}$. This is given by

$$
\begin{aligned}
\frac{d \mathbf{v}}{d \operatorname{vec}^{\top} \boldsymbol{\Phi}}= & \left(\sigma^{2} \mathbf{I}_{s}-\boldsymbol{\Phi}^{\top} \boldsymbol{\Phi}+\frac{\sigma^{2}}{2}\left(\mathbf{v}^{\boldsymbol{\top}} \otimes \mathbf{v}\right)+\frac{\sigma^{2}}{2} \mathbf{v} \mathbf{v}^{\top}\right)^{-1} \\
& \times\left[\left(\mathbf{v}^{\boldsymbol{\top} \otimes} \mathbf{I}_{s}\right)-\left(\mathbf{v}^{\boldsymbol{\top}} \otimes \mathbf{v} \mathbf{v}^{\boldsymbol{\top}}\right)\right]\left[\left(\boldsymbol{\Phi}^{\top} \otimes \mathbf{I}\right) \mathbf{P}+\left(\mathbf{I} \otimes \boldsymbol{\Phi}^{\top}\right)\right]
\end{aligned}
$$

where $\mathbf{P}$ is the vec-permutation matrix (Henderson and Searle, 1981); see Appendix B.2 for the derivation. The sensitivity of the optimal perturbation is then

$$
\frac{d \mathbf{v}(t)}{d \boldsymbol{\theta}^{\top}}=\frac{d \mathbf{v}(t)}{d \operatorname{vec} \boldsymbol{\Phi}(t)} \frac{d \operatorname{vec} \boldsymbol{\Phi}(t)}{d \operatorname{vec}^{\top} \mathbf{M}} \frac{d \operatorname{vec} \mathbf{M}}{d \boldsymbol{\theta}^{\top}}
$$

where $d \operatorname{vec} \boldsymbol{\Phi}(t) / d \operatorname{vec}^{\top} \mathbf{M}$ is given by (43) for continuous systems and by (44) for discrete systems, and $d \operatorname{vec} \mathbf{M} / d \boldsymbol{\theta}^{\top}$ is given by (37).

\subsection{Anisotropic measures of transient amplification}

In Section 1.1 the reactivity, amplification envelope, and optimal perturbation are defined in terms of the Euclidean distance from the equilibrium $\hat{\mathbf{x}}$. In applications, some components of the perturbation may be of more interest than others. For example, one might want apply relative weights to the elements of the perturbation vector, to translate numbers of individuals into biomass or nutrient content. The transient dynamics of such anisotropic state vectors are easily analyzed.

To apply weights to the elements of the perturbation, define a diagonal weighting kernel matrix $\mathbf{K}$, whose elements give the relative importance of each element of $\mathbf{z}$. Define the rescaled vector $\tilde{\mathbf{z}}=\mathbf{K} \mathbf{z}$, the norm of which is $\|\tilde{\mathbf{z}}\|=\left(\mathbf{z}^{\top} \mathbf{K}^{2} \mathbf{z}\right)^{1 / 2}$. Solving the optimization problem for $\|\tilde{\mathbf{z}}\|$, we obtain a modified expression for the reactivity and the amplification envelope; these are the same as (7) and (15) but with the Jacobian and fundamental matrix 
replaced by

$$
\begin{aligned}
\tilde{\mathbf{M}} & =\mathbf{K M K}^{-1} \\
\tilde{\mathbf{\Phi}} & =\mathbf{K} \mathbf{\Phi} \mathbf{K}^{-1}
\end{aligned}
$$

Differentiating these expressions gives

$$
\frac{d \operatorname{vec} \tilde{\mathbf{M}}}{d \operatorname{vec}^{\top} \mathbf{M}}=\frac{d \operatorname{vec} \tilde{\boldsymbol{\Phi}}}{d \operatorname{vec}^{\top} \boldsymbol{\Phi}}=\left(\mathbf{K}^{-1} \otimes \mathbf{K}\right)
$$

Then, for any transient index $r$, we can write

$$
\frac{d r}{d \boldsymbol{\theta}^{\top}}=\frac{d r}{d \operatorname{vec}^{\top} \tilde{\boldsymbol{\Phi}}} \frac{d \tilde{\boldsymbol{\Phi}}}{d \operatorname{vec}^{\top} \boldsymbol{\Phi}} \frac{d \operatorname{vec} \boldsymbol{\Phi}}{d \operatorname{vec}^{\top} \tilde{\mathbf{M}}} \frac{d \operatorname{vec} \tilde{\mathbf{M}}}{d \operatorname{vec}^{\top} \mathbf{M}} \frac{d \operatorname{vec} \mathbf{M}}{d \boldsymbol{\theta}^{\top}}
$$

The weighting kernel effectively "stretches" the space in which we measure deviations from equilibrium. The same result could be obtained by scaling the nonlinear model, and then analyzing the perturbations in the rescaled space. The advantage of the weighting kernel is that it allows for consideration of different norms without the need to repeat the calculations of sensitivity matrices.

\section{Applications to consumer-resource dynamics}

Consumer-resource models are often reactive, and reactivity tends to increase with the number of species (Chen and Cohen, 2001; Neubert et al, 2004). In this section, we apply sensitivity analysis to a predator-prey model and a multispecies food web model. The results provide insight into the mechanisms by which reactivity and transient amplification can be produced. 


\subsection{Predator-prey interactions}

The Rosenzweig-MacArthur model (Rosenzweig and MacArthur, 1963) for a predator $(Z)$ and prey $(P)$ is

$$
\begin{aligned}
\frac{d P}{d t} & =r P\left(1-\frac{P}{K}\right)-g \frac{P}{P+P_{h}} Z \\
\frac{d Z}{d t} & =\epsilon g \frac{P}{P+P_{h}} Z-\delta Z
\end{aligned}
$$

The prey grows logistically, with maximum growth rate $r$ and carrying capacity $K$. The predator has a mortality rate $\delta$ and exhibits a Holling type II functional response; $g$ is the maximum predation rate, $P_{h}$ the half-saturation prey density, and $\epsilon$ the assimilation efficiency.

The system has a single coexistence equilibrium, given by

$$
\begin{aligned}
\hat{P} & =\frac{P_{h} \delta}{\epsilon g-\delta} \\
\hat{Z} & =r\left(1-\frac{\hat{P}}{K}\right) \frac{\hat{P}+P_{h}}{g}
\end{aligned}
$$

The model (52-53) can be expressed in the formalism (1), with ${ }^{2}$

$$
\mathbf{A}=\left[\begin{array}{cc}
r\left(1-\frac{P}{K}\right)-\frac{g}{P+P_{h}} Z & 0 \\
0 & \epsilon \frac{g P}{P+P_{h}}-\delta
\end{array}\right]
$$

We define a population vector $\mathbf{x} \equiv[P Z]^{\top}$ and a parameter vector $\boldsymbol{\theta} \equiv\left[\epsilon g \delta P_{h} r K\right]^{\top}$. The first derivatives of $\mathbf{A}$ are

$$
\mathbf{B} \equiv \frac{\partial \operatorname{vec} \mathbf{A}}{\partial \mathbf{x}^{\top}}=\left[\begin{array}{cc}
\frac{-r}{K}+\frac{g}{\left(P+P_{h}\right)^{2}} Z & \frac{-g}{P+P_{h}} \\
0 & 0 \\
0 & 0 \\
\epsilon \frac{g P_{h}}{\left(P+P_{h}\right)^{2}} & 0
\end{array}\right]
$$

\footnotetext{
${ }^{2}$ It is not essential to write $\mathbf{A}$ as a diagonal matrix, but in this case it simplifies the calculation of derivatives.
} 


$$
\frac{\partial \operatorname{vec} \mathbf{A}}{\partial \boldsymbol{\theta}^{\top}}=\left[\begin{array}{cccccc}
0 & \frac{-Z}{P+P_{h}} & 0 & \frac{g}{\left(P+P_{h}\right)^{2}} Z & 1-\frac{P}{K} & \frac{-r P}{K^{2}} \\
0 & 0 & 0 & 0 & 0 & 0 \\
0 & 0 & 0 & 0 & 0 & 0 \\
\frac{g P}{\left(P+P_{h}\right)} & \frac{\epsilon P}{\left(P+P_{h}\right)} & -1 & \frac{-\epsilon g P}{\left(P+P_{h}\right)^{2}} & 0 & 0
\end{array}\right]
$$

The second derivatives are

$$
\begin{aligned}
& \frac{\partial \mathrm{vec} \mathbf{B}}{\partial \mathbf{x}^{\top}}=\left[\begin{array}{cc}
-2 \frac{g}{\left(P+P_{h}\right)^{3}} Z & \frac{g}{\left(P+P_{h}\right)^{2}} \\
0 & 0 \\
0 & 0 \\
-2 \epsilon \frac{g P_{h}}{\left(P+P_{h}\right)^{3}} & 0 \\
\frac{g}{\left(P+P_{h}\right)^{2}} & 0 \\
0 & 0 \\
0 & 0 \\
0 & 0
\end{array}\right] \\
& \frac{\partial \operatorname{vec} \mathbf{B}}{\partial \boldsymbol{\theta}^{\top}}=\left[\begin{array}{cccccc}
0 & \frac{Z}{\left(P+P_{h}\right)^{2}} & 0 & -2 \frac{g}{\left(P+P_{h}\right)^{3}} Z & \frac{-1}{K} & \frac{r}{K^{2}} \\
0 & 0 & 0 & 0 & 0 & 0 \\
0 & 0 & 0 & 0 & 0 & 0 \\
\frac{g P}{\left(P+P_{h}\right)^{2}} & \frac{\epsilon P}{\left(P+P_{h}\right)^{2}} & 0 & \frac{\epsilon g\left(P-P_{h}\right)}{\left(P+P_{h}\right)^{3}} & 0 & 0 \\
0 & \frac{-1}{\left(P+P_{h}\right)} & 0 & \frac{g}{\left(P+P_{h}\right)^{2}} & 0 & 0 \\
0 & 0 & 0 & 0 & 0 & 0 \\
0 & 0 & 0 & 0 & 0 & 0 \\
0 & 0 & 0 & 0 & 0 & 0
\end{array}\right]
\end{aligned}
$$

We will examine two equilibria, with parameters given in Table 1; both are locally stable.

We focus first on equilibrium $A$.

\subsubsection{Transient growth following arbitrary perturbations}

Figure 1 illustrates the transient growth of perturbations around equilibrium $A$. In the linear approximation, deviations from the equilibrium evolve according to (3). We consider initial conditions on the unit circle centered on the equilibrium, shown as gray dots in Figure 1; the transient responses corresponding to those initial conditions are shown as black dots.

States outside the unit circle at any time (e.g., Figure 1a) correspond to initial perturbations that are amplified. The maximum amplification occurs at $t=5.7$ (Figure 1b). 
Perturbations then decay (Figure 1c) as the system returns to equilibrium. The direction

of the optimal perturbation at each time is shown by a gray arrow (positive and negative perturbations have the same effect due to symmetry of the linearized system). The optimal perturbation rotates clockwise between $t=2$ and $t=5.7$. The largest growth at $t=5.7$ results from a perturbation of the predator population only; this suggests a "predator-driven" mechanism for reactive dynamics around this equilibrium.

The amplification envelope (Figure 2a) shows that maximum amplification occurs at $t=5.7$. The initial rate of growth is the reactivity $\left(\nu_{0}\right)$. Fluctuations in the amplification envelope reflect damped oscillations in the dynamics of the perturbed system.

Sensitivity of the amplification envelope, calculated from (45) is shown as a function of time in Figure 2b. The sensitivities exhibit synchronous fluctuations that appear to be modulated by the amplification itself. For some parameters, the sensitivity changes sign during the period of oscillation. There also appears to be a qualitative change in the effect of parameters after the first minimum of the amplification envelope, suggesting a change in dynamics between the initial growth and the period of decay.

The elasticities of reactivity and of the amplification envelope at the time of maximum growth are shown in Figure 3. Increases in the predation rate, mortality rate, or carrying capacity will increase reactivity, whereas the assimilation efficiency, half-saturation prey density, and prey growth rate have the opposite effect. This information can be used to explore the mechanisms regulating transient dynamics.

\subsubsection{Mechanisms of amplification}

Sensitivity analysis can identify the ecological processes responsible for transient amplification. In this model there appear to be two distinct mechanisms leading to reactive dynamics; we describe them as "predator-driven" and "prey-driven" mechanisms. We carry out this analysis in terms of the dimensionless parameters $\epsilon$ (the assimilation efficiency of the predator), $g / r$ (the scaled predation rate), $\delta / r$ (the scaled mortality rate), and $K / P_{h}$ (the prey enrichment ratio).

We rewrite the predator-prey model in terms of these scaled variables, and compute the sensitivities following the same procedure as before, with the parameter $\boldsymbol{\theta}$ now containing 
the four dimensionless parameters.

First, we examine the effect of assimilation and predation. Reactivity is plotted in Figure 4 in the $(\epsilon, g / r)$ plane in the region where coexistence is a stable equilibrium. There are two distinct regions of high reactivity; one corresponds to low predation rate but high efficiency and the other corresponds to high predation rate but low efficiency. Intermediate values of $\epsilon$ and $g / r$ lead to low reactivity. To describe the mechanisms driving transient growth, we will focus on the equilibrium points $A$ and $B$ (Table 1); these have the same reactivity, but differ in other properties.

The sign of the sensitivity of reactivity reveals some differences between the two reactive regions (Figure 5). Predation and enrichment have positive effects on reactivity, except where reactivity is very small. Assimilation and mortality can have a positive or negative effect, depending on the parameter values.

Reactivity at equilibrium $A$ is increased by reducing the assimilation efficiency and increasing the mortality rate. This implies that the mechanism for amplification relies on slow uptake of resources by the predator. At equilibrium, a relatively small predator population keeps the prey constant; consequently, a small reduction in the predator population translates into rapid multiplication of the prey. We call this predator-driven transient dynamics. Eventually, the predator catches up and removes the excess prey; this explains the qualitative change in sensitivity of the amplification envelope for $t>11$ (Figure $2 \mathrm{~b}$ ).

In contrast, at equilibrium $B$ there is a prey-driven transient response. At equilibrium, a large predator population is sustained by the highly efficient, albeit slow, consumption of prey. The predators can take advantage of transient increases in the prey. Because amplification at equilibrium $B$ relies on rapid predator growth, reactivity is enhanced by increasing assimilation efficiency and reducing mortality rate.

The transition between these two scenarios is accompanied by a change in the ratio of predator to prey biomass. The dashed white line in Figure 4 bounds the region of resourcedepleted equilibria (above the curve), where transient dynamics are controlled by the lower species in the food chain.

These mechanisms are also reflected in the directions of the optimal initial perturbations and the resulting responses, as shown in Figure 6 for a fixed time $(t=4)$ during the initial 
period of growth. Perturbations leading to maximal growth in the predator-driven scenario correspond to a decrease in predator biomass and increase in prey or, by symmetry, increase in predator and decrease in prey. The transient response also lies in the second and fourth quadrants (Figure 6a). In the prey-driven scenario, by contrast, optimal perturbations and their associated transient response are found in the first and third quadrants; they correspond to concurrent increase or reduction in the prey and predator biomass (Figure $6 \mathrm{~b})$.

\subsection{Multiple food chains}

Armstrong (1994) introduced a model for a size-structured marine food web. The model includes size classes of phytoplankton $\left(P_{1}, P_{2}, \ldots\right)$ and zooplankton $\left(Z_{1}, Z_{2}, \ldots\right)$. We consider the case of connected food chains, where each zooplankton size class feeds on phytoplankton in the corresponding size class and on zooplankton in the size class below (Figure 7). Allometric relations are assumed for the size-dependence of biological activities.

In our model, we assume logistic growth of phytoplankton, with a carrying capacity chosen to produce the same equilibrium as in Armstrong (1994; his case $T=5)$ for the case $N_{T}=5$. Using the parameters in Table 2 (the values are taken from Armstrong, 1994), the model allows for coexistence of 3 phytoplankton classes and 5 zooplankton classes.

The equations are

$$
\begin{aligned}
\frac{d P_{i}}{d t} & =\left[r_{i}\left(1-\frac{\sum P}{K}\right)-\lambda-Z_{i} \frac{g_{i}}{P_{s}}\right] P_{i} \\
\frac{d Z_{i}}{d t} & =\left[\epsilon \frac{g_{i}}{P_{s}}\left(P_{i}+Z_{i-1}\right)-\delta_{i}-Z_{i+1} \frac{g_{i+1}}{P_{s}}\right] Z_{i}
\end{aligned}
$$

with $i=0, \ldots, 4$.

The predation rate $g_{i}$, mortality rate $\delta_{i}$, and prey growth rate $r_{i}$ depend on the body length $L_{i}$ according to the allometric relations:

$$
\begin{aligned}
g_{i} & =g_{0}\left(\frac{L_{i}}{L_{0}}\right)^{\beta} \\
\delta_{i} & =\delta_{0}\left(\frac{L_{i}}{L_{0}}\right)^{\beta}+\lambda\left(\frac{L_{i}}{L_{0}}\right)^{\gamma}
\end{aligned}
$$




$$
\begin{aligned}
r_{i} & =r_{0}\left(\frac{L_{i}}{L_{0}}\right)^{\beta} \\
L_{i} & =4^{i} \times L_{0}
\end{aligned}
$$

Values for $\beta$ and $\gamma$ are given in Table 2 .

The unique coexistence equilibrium is shown in Figure 8. In this food web, the total predator biomass exceeds the prey biomass because of the rapid turnover rate of phytoplankton.

For sensitivity analysis, we write the model in matrix form with a state vector, of length

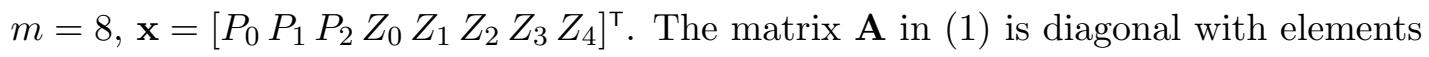

$$
\begin{aligned}
& A_{11}=r_{0}\left(1-\frac{P_{0}+P_{1}+P_{2}}{K}\right)-\lambda-Z_{0} \frac{g_{0}}{P_{s}} \\
& A_{22}=r_{0} 4^{\beta}\left(1-\frac{P_{0}+P_{1}+P_{2}}{K}\right)-\lambda-Z_{1} \frac{g_{0} 4^{\beta}}{P_{s}} \\
& A_{33}=r_{0} 4^{2 \beta}\left(1-\frac{P_{0}+P_{1}+P_{2}}{K}\right)-\lambda-Z_{2} \frac{g_{0} 4^{2 \beta}}{P_{s}} \\
& A_{44}=\epsilon \frac{g_{0}}{P_{s}} P_{1}-\delta_{0}-\lambda-Z_{1} \frac{g_{0} 4^{\beta}}{P_{s}} \\
& A_{55}=\epsilon \frac{g_{0} 4^{\beta}}{P_{s}}\left[\left(P_{1}+Z_{0}\right]-\delta_{0} 4^{\beta}-\lambda 4^{\gamma}-Z_{2} \frac{g_{0} 4^{2 \beta}}{P_{s}}\right. \\
& A_{66}=\epsilon \frac{g_{0} 4^{2 \beta}}{P_{s}}\left[P_{2}+Z_{1}\right]-\delta_{0} 4^{2 \beta}-\lambda 4^{2 \gamma}-Z_{3} \frac{g_{0} 4^{3 \beta}}{P_{s}} \\
& A_{77}=\epsilon \frac{g_{0} 4^{3 \beta}}{P_{s}} Z_{2}-\delta_{0} 4^{3 \beta}-\lambda 4^{3 \gamma}-Z_{4} \frac{g_{0} 4^{4 \beta}}{P_{s}} \\
& A_{88}=\epsilon \frac{g_{0} 4^{4 \beta}}{P_{s}} Z_{3}-\delta_{0} 4^{4 \beta}-\lambda 4^{4 \gamma}
\end{aligned}
$$

The matrices $d \operatorname{vec} \mathbf{A} / d \mathbf{x}^{\top}, d \operatorname{vec} \mathbf{A} / d \boldsymbol{\theta}^{\top}$, and $d \operatorname{vec} \mathbf{B} / d \boldsymbol{\theta}^{\top}$ required for the sensitivity analysis are large, but because $\mathbf{A}$ is diagonal, most of their entries are zero. These matrices are presented in Verdy (2008).

The equilibrium is reactive, with the maximum amplification occurring at $t=37$ (Figure 9). Time is measured in days in this parameterization, so the largest effect of perturbing the equilibrium appears more than a month later. The amplification envelope remains above 1 for over 3 years $(t=1185$ days $)$. This is an example of a system in which transient amplification is likely to be more ecologically relevant than the asymptotic return to the 
equilibrium.

Even in the 2-species predator-prey model, the effects of the parameters were not always intuitive. In more complex models, sensitivity analysis is particularly useful for understanding what controls the transient dynamics and how a system responds to changes in the parameters. In this food web model, the assimilation efficiency, predation rate, prey growth rate and carrying capacity have positive impacts on reactivity, whereas mortality and saturation prey density have negative impacts (Figure 10a). Parameters that increase reactivity are found to increase the maximum amplification as well (Figure 10b), an indication that the same mechanism driving amplification at $t=0$ causes the maximum growth at $t=37$. The elasticity to assimilation efficiency is particularly large: a $10 \%$ increase in $\epsilon$ would increase reactivity by more than $20 \%$ and the maximum amplification by more than $70 \%$.

Sensitivity analysis could be employed to investigate, for example, how the mechanisms of transient dynamics vary with food chains' lengths. In Armstrong's (1994) model, the number of trophic levels is controlled by the total nutrient availability. Although this question is not addressed here, it could be studied using the framework presented in this paper.

\section{Discussion}

When perturbed from their equilibria, ecosystems exhibit transient dynamics. These transients can last for extended periods of time and have important ecological consequences. When the equilibrium is reactive, small fluctuations can be amplified. In nature, perturbations result from the stochasticity inherent to the environment and the biological processes themselves. Ecologists have long conceptualized ecological stability in terms of the asymptotic rate of return to equilibrium [see Ives and Carpenter (2007) for a recent example], but it is not yet widely appreciated how deficient this approach is. It bears repeating that the asymptotic rate of return does not determine the transient amplification. For example, it is easy to construct matrices with identical eigenvalues, and hence identical asymptotic rates of return, but with very different reactivities and amplification envelopes (e.g., Trefethen et 
al, 1993; Neubert and Caswell, 1997).

As more examples accumulate of reactive ecological models, it becomes of more interest to understand how the transient responses depend on the parameters of the underlying model. The sensitivity analyses we have presented here make it possible to do so. Sensitivity analysis, in this context, can serve two purposes. One is to reveal the biological mechanisms that produce it. Knowing, for example, that reactivity in the size-structured food web model is highly elastic to assimilation efficiency suggests that the amplification of perturbations is related to the efficiency of energy transfer between trophic levels. The second is predicting the effect of parameter changes, such as might occur due to pollution, nutrient input, climate change, or other events. Of particular interest would be analysis of infectious disease outbreaks and biological invasions, in both of which transient phenomena are particularly important.

Writing the model in matrix form as in Eqs. (1-2) focuses attention on the role of the per-capita (or per-unit) rates that appear in the matrix $\mathbf{A}$, and the dependence of those rates on the parameters and the population state. Our analysis, however, can also be applied to systems not written as matrix models, in which (1) and (2) are replaced by

$$
\begin{aligned}
\frac{d \mathbf{x}}{d t} & =\mathbf{f}[\boldsymbol{\theta}, \mathbf{x}] \\
\mathbf{x}(t+1) & =\mathbf{f}[\boldsymbol{\theta}, \mathbf{x}(t)]
\end{aligned}
$$

where $\mathbf{f}[\boldsymbol{\theta}, \mathbf{x}]$ is a vector-valued function. The linearization of (63) or (64) around an equilibrium $\hat{\mathbf{x}}$ is given by

$$
\mathbf{M}=\left.\frac{d \mathbf{f}}{d \mathbf{x}}\right|_{\hat{\mathbf{x}}}
$$

This linearization, obtained without benefit of the matrix formulation, can then be further analyzed as described here. In particular, we obtain for its sensitivity

$$
\frac{d \operatorname{vec} \mathbf{M}}{d \boldsymbol{\theta}^{\top}}=\frac{\partial \operatorname{vec} \mathbf{M}}{\partial \boldsymbol{\theta}^{\top}}-\frac{\partial \operatorname{vec} \mathbf{M}}{\partial \mathbf{x}^{\top}} \mathbf{M}^{-1} \frac{\partial \mathbf{f}}{\partial \boldsymbol{\theta}^{\top}}
$$

which would then be used in Eqs. (40), (45), and (47). The remainder of the sensitivity calculations proceed unchanged. 


\section{Acknowledgments}

Financial support provided by NSF grant DEB-0343820, NOAA grant NA03-NMF4720491, the Ocean Life Institute of the Woods Hole Oceanographic Institution, and the Academic Programs Office of the MIT-WHOI Joint Program in Oceanography. We thank M. Neubert, G. Flierl, and an anonymous reviewer for discussion and comments.

\section{References}

Armstrong, R. A., 1994. Grazing limitation and nutrient limitation in marine ecosystems - steady-state solutions of an ecosystem model with multiple food-chains. Limnol. Oceanogr., 39: 597-608.

Caswell, H., 2001. Matrix population models: Construction, Analysis, and Interpretation 2nd ed. Sinauer Associates, Sunderland, Massachusetts, USA.

Caswell, H., 2007. Sensitivity analysis of transient population dynamics. Ecol. Lett., 10: $1-15$.

Caswell, H., 2008. Perturbation analysis of nonlinear matrix population models. Demogr. Res., 18: 59-116.

Caswell, H., and M. G. Neubert, 2005. Reactivity and transient dynamics of discrete-time ecological systems. J. Differ. Equ. Appl., 11: 295-310.

Chen, X., and J. E. Cohen, 2001. Transient dynamics and food-web complexity in the lotka-volterra cascade model. Proc. R. Soc. Lond. Ser. B-Biol. Sci., 268: 869-877.

Coddington, E. A., and N. Levinson, 1955. Theory of ordinary differential equations. McGraw-Hill, New York, USA.

Farrell, B. F., and P. J. Ioannou, 1996. Generalized stability theory .1. autonomous operators. J. Atmos. Sci., 53: 2025-2040.

Hastings, A., 2004. Transients: the key to long-term ecological understanding? TREE, 19: $39-45$.

Henderson, H. V., and S. R. Searle, 1981. The vec-permutation matrix, the vec operator and kronecker products: a review. Lin. Multilin. Algebra, 9: 271-288.

Ives, A. R., and S. R. Carpenter, 2007. Stability and diversity of ecosystems. Science, 317: $58-62$.

Magnus, J. R., and H. Neudecker, 1985. Matrix differential calculus with applications to simple, hadamard, and kronecker products. J. Math. Psychol., 29: 474-492. 
Magnus, J. R., and H. Neudecker, 1988. Matrix Differential Calculus with Applications in Statistics and Econometrics. John Wiley \& Sons, New York, USA.

Marvier, M., P. Kareiva, and M. G. Neubert, 2004. Habitat destruction, fragmentation, and disturbance promote invasion by habitat generalists in a multispecies metapopulation. Risk Anal., 24: 869-878.

May, R. M., 1973. Stability and complexity in model ecosystems. Princeton University Press, Princeton, New Jersey, USA.

Nel, D. G., 1980. On matrix differentiation in statistics. S. Afr. Stat. J., 14: 137-193.

Neubert, M. G., and H. Caswell, 1997. Alternatives to resilience for measuring the responses of ecological systems to perturbations. Ecology, 78: 653-665.

Neubert, M. G., H. Caswell, and J. D. Murray, 2002. Transient dynamics and pattern formation: reactivity is necessary for turing instabilities. Math. Biosci., 175: 1-11.

Neubert, M. G., T. Klanjscek, and H. Caswell, 2004. Reactivity and transient dynamics of predator-prey and food web models. Ecol. Model., 179: 29-38.

Pimm, S. L., 1984. The complexity and stability of ecosystems. Nature, 307: 321-326.

Rosenzweig, M. L., and R. H. MacArthur, 1963. Graphical representation and stability conditions of predator-prey interactions. Am. Nat., 97: 209-223.

Roth, W. E., 1934. On direct product matrices. Bull. Am. Math. Soc., 40: 461-468.

Stewart, G. W., 1991. Perturbation theory for the singular value decomposition. SVD and Signal Processing, II: Algorithms, Analysis and Applications, R. J. Vaccaro, Ed., Elsevier, Amsterdam, pp. 99-109.

Trefethen, L. N., 1992. Pseudospectra of matrices. Numerical Analysis 1991, Proc. 14th Dundee Conf., Longman Scientific and Technical,, D. F. Griffiths and G. A. Watson, Eds., Essex, UK, pp. 234-266.

Trefethen, L. N., and M. Embree, 2005. Spectra and Pseudospectra: The Behavior of nonnormal matrices and operators. Princeton University Press, Princeton, New Jersey, USA.

Trefethen, L. N., A. E. Trefethen, S. C. Reddy, and T. A. Driscoll, 1993. Hydrodynamic stability without eigenvalues. Science, 261: 578-584.

Verdy, A., 2008. Dynamics of marine zooplankton: social behavior, ecological interactions, and physically-induced variability, Ph.D. thesis, MIT-WHOI Joint Program in Oceanography. 


\section{A Sensitivity of stable equilibria}

The sensitivity of the equilibrium for the discrete model is derived in Caswell (2008). Here we show the derivation for the continuous model (1). The equilibrium $\hat{\mathbf{x}}$ satisfies

$$
0=\mathbf{A}[\boldsymbol{\theta}, \hat{\mathbf{x}}(\boldsymbol{\theta})] \hat{\mathbf{x}}
$$

Taking the differential of both sides gives

$$
0=(d \mathbf{A}) \hat{\mathbf{x}}+\mathbf{A}(d \hat{\mathbf{x}})
$$

Applying the vec operator to both sides, noting that vec $\hat{\mathbf{x}}=\hat{\mathbf{x}}$, and using Roth's (1934) relation (22), gives

$$
0=\left(\hat{\mathbf{x}}^{\top} \otimes \mathbf{I}_{s}\right) d \operatorname{vec} \mathbf{A}+\mathbf{A} d \hat{\mathbf{x}}
$$

But $\mathbf{A}$ depends on both $\boldsymbol{\theta}$ and $\hat{\mathbf{x}}$, so

$$
0=\left(\hat{\mathbf{x}}^{\top} \otimes \mathbf{I}_{s}\right)\left(\frac{\partial \operatorname{vec} \mathbf{A}}{\partial \boldsymbol{\theta}} d \boldsymbol{\theta}+\frac{\partial \mathbf{A}}{\partial \mathbf{x}^{\top}} d \hat{\mathbf{x}}\right)+\mathbf{A} d \hat{\mathbf{x}}
$$

Collecting terms, solving for $d \hat{\mathbf{x}}$, and rearranging gives

$$
d \hat{\mathbf{x}}=\left(-\mathbf{A}-\left(\hat{\mathbf{x}} \otimes \mathbf{I}_{s}\right) \frac{\partial \mathrm{vec} \mathbf{A}}{\partial \mathbf{x}^{\top}}\right)^{-1}\left(\hat{\mathbf{x}}^{\top} \otimes \mathbf{I}_{s}\right) \frac{\partial \mathbf{A}}{\partial \boldsymbol{\theta}^{\top}} d \boldsymbol{\theta}
$$

The "first identification theorem" of Magnus and Neudecker (1985) says that the matrix multiplying $d \boldsymbol{\theta}$ is the derivative $d \hat{\mathbf{x}} / d \boldsymbol{\theta}^{\top}$, as shown in (29).

\section{B Sensitivities of eigenvalues and singular vectors}

\section{B.1 Sensitivity of eigenvalues}

The sensitivity of reactivity in continuous systems requires the sensitivity of the largest eigenvalue $\nu_{0}$ of $\mathbf{H}=\left(\mathbf{M}+\mathbf{M}^{\top}\right) / 2$. Let $\mathbf{w}$ be the right eigenvector of $\mathbf{H}$ corresponding to $\nu_{0}$. Since $\mathbf{H}$ is symmetric, $\mathbf{w}$ is also the left eigenvector, and we suppose that $\mathbf{w}^{\top} \mathbf{w}=1$. 
The sensitivity of $\nu_{0}$ to $\mathbf{M}$ is

$$
\frac{d \nu_{0}}{d \operatorname{vec}^{\top} \mathbf{M}}=\frac{d \nu_{0}}{d \operatorname{vec}^{\top} \mathbf{H}} \frac{d \operatorname{vec} \mathbf{H}}{d \operatorname{vec}^{\top} \mathbf{M}}
$$

where the standard eigenvalue sensitivity formula, written in matrix calculus notation, is

$$
\frac{d \nu_{0}}{d \operatorname{vec}^{\top} \mathbf{H}}=\mathbf{w}^{\top} \otimes \mathbf{w}^{\top}
$$

The sensitivity of $\mathbf{H}$ to $\mathbf{M}$ is

$$
\frac{d \operatorname{vec} \mathbf{H}}{d \operatorname{vec}^{\top} \mathbf{M}}=\frac{1}{2}\left(\mathbf{I}_{s^{2}}+\mathbf{P}\right)
$$

where $\mathbf{P}$ is the vec-permutation matrix (Henderson and Searle, 1981). Because both $\mathbf{H}$ and $\mathbf{P}$ are symmetric in this case, it follows that

$$
\frac{d \nu_{0}}{d \operatorname{vec}^{\top} \mathbf{H}} \mathbf{P}=\frac{d \nu_{0}}{d \operatorname{vec}^{\top}\left(\mathbf{H}^{\top}\right)}=\frac{d \nu_{0}}{d \operatorname{vec}^{\top} \mathbf{H}}
$$

Thus

$$
\frac{d \nu_{0}}{d \operatorname{vec}^{\top} \mathbf{M}}=\mathbf{w}^{\top} \otimes \mathbf{w}^{\top}
$$

\section{B.2 Sensitivity of singular vectors}

We treat the right singular vector $\mathbf{v}$ of a matrix $\mathbf{X}$, corresponding to a singular value $\sigma$, as the eigenvector $\mathbf{v}$ of $\mathbf{X}^{\top} \mathbf{X}$ corresponding to the eigenvalue $\sigma^{2}$, normalized to unit length. We find the derivative of $\mathbf{v}$ using by adapting the approach used for eigenvectors of population projection matrices by Caswell (2008). Let $\mathbf{Y}=\mathbf{X}^{\top} \mathbf{X}$. Then $\mathbf{Y} \mathbf{v}=\sigma^{2} \mathbf{v}$ and $\mathbf{v}$ is also a fixed point of the system

$$
\mathbf{v}(t+1)=\frac{\mathbf{Y} \mathbf{v}(t)}{\|\mathbf{Y} \mathbf{v}(t)\|}
$$

Write this fixed point as

$$
\mathbf{v}=\frac{\mathbf{Y} \mathbf{v}}{\left(\mathbf{v}^{\top} \mathbf{Y}^{\top} \mathbf{Y} \mathbf{v}\right)^{1 / 2}}
$$

and differentiate, obtaining

$$
d \mathbf{v}=\frac{\mathbf{Y}(d \mathbf{v})+(d \mathbf{Y}) \mathbf{v}}{\left(\mathbf{v}^{\top} \mathbf{Y}^{\top} \mathbf{Y} \mathbf{v}\right)^{1 / 2}}-\frac{\mathbf{Y} \mathbf{v} d\left(\mathbf{v}^{\top} \mathbf{Y}^{\top} \mathbf{Y} \mathbf{v}\right)}{2\left(\mathbf{v}^{\top} \mathbf{Y}^{\top} \mathbf{Y} \mathbf{v}\right)^{3 / 2}}
$$


Expand the differential in the second term and then simplify, using the relations

$$
\begin{aligned}
\mathbf{Y} \mathbf{v} & =\mathbf{Y}^{\top} \mathbf{v}=\sigma^{2} \mathbf{v} \\
\mathbf{v}^{\top} \mathbf{Y}^{\top} & =\mathbf{v}^{\top} \mathbf{Y}=\sigma^{2} \mathbf{v}^{\top} \\
\mathbf{v}^{\top} \mathbf{v} & =1 \\
\mathbf{v}^{\top} \mathbf{Y}^{\top} \mathbf{Y} \mathbf{v} & =\sigma^{4}
\end{aligned}
$$

Applying the vec operator yields, after some rearrangement

$$
\left(\sigma^{2} \mathbf{I}_{s}-\mathbf{Y}+\frac{\sigma^{2}}{2}\left(\mathbf{v}^{\top} \otimes \mathbf{v}\right)+\frac{\sigma^{2}}{2} \mathbf{v v}^{\top}\right) d \mathbf{v}=\left[\left(\mathbf{v}^{\top} \otimes \mathbf{I}_{s}\right)-\left(\mathbf{v}^{\boldsymbol{\top}} \otimes \mathbf{v} \mathbf{v}^{\top}\right)\right] d \operatorname{vec} \mathbf{Y}
$$

Since $\mathbf{Y}=\mathbf{X}^{\top} \mathbf{X}$, it follows that

$$
d \operatorname{vec} \mathbf{Y}=\left[\left(\mathbf{X}^{\top} \otimes \mathbf{I}\right) \mathbf{P}+\left(\mathbf{I} \otimes \mathbf{X}^{\top}\right)\right] d \operatorname{vec} \mathbf{X}
$$

where $\mathbf{P}$ is the vec-permutation matrix. Finally,

$$
\begin{aligned}
\frac{d \mathbf{v}}{d \operatorname{vec}^{\top} \mathbf{X}}= & \left(\sigma^{2} \mathbf{I}_{s}-\mathbf{X}^{\top} \mathbf{X}+\frac{\sigma^{2}}{2}\left(\mathbf{v}^{\top} \otimes \mathbf{v}\right)+\frac{\sigma^{2}}{2} \mathbf{v} \mathbf{v}^{\top}\right)^{-1} \\
& \times\left[\left(\mathbf{v}^{\top} \otimes \mathbf{I}_{s}\right)-\left(\mathbf{v}^{\top} \otimes \mathbf{v} \mathbf{v}^{\top}\right)\right]\left[\left(\mathbf{X}^{\top} \otimes \mathbf{I}\right) \mathbf{P}+\left(\mathbf{I} \otimes \mathbf{X}^{\top}\right)\right]
\end{aligned}
$$


Table 1: Parameters for the predator-prey model

\begin{tabular}{lllll}
\hline \hline \multirow{2}{*}{ Symbol } & Definition & Value & \multirow{2}{*}{ Units } \\
\cline { 3 - 4 } & & Equilibrium A & Equilibrium B & \\
\hline$\epsilon$ & assimilation efficiency & 0.15 & 0.8 & - \\
$g$ & predation rate & 2.3 & 0.8 & $\mathrm{~d}^{-1}$ \\
$\delta$ & mortality rate & 0.1 & 0.1 & $\mathrm{~d}^{-1}$ \\
$P_{h}$ & half-saturation prey density & 1 & 1 & $\mu \mathrm{mol} \mathrm{m}^{-3}$ \\
$r$ & prey growth rate & 1 & 1 & $\mathrm{~d}^{-1}$ \\
$K$ & carrying capacity & 1.25 & 1.25 & $\mu \mathrm{mol} \mathrm{m}^{-3}$ \\
$\hat{P}$ & equilibrium prey biomass & 0.41 & 0.18 & $\mu \mathrm{mol} \mathrm{m}^{-3}$ \\
$\hat{Z}$ & equilibrium predator biomass & 0.41 & 1.26 & $\mu \mathrm{mol} \mathrm{m}^{-3}$ \\
\hline \hline
\end{tabular}

Table 2: Parameters for the multiple food chains model; values are from Armstrong (1994).

\begin{tabular}{llll}
\hline \hline Symbol & Definition & Value & Units \\
\hline$L_{0}$ & length of organism in smallest size class & 1 & (arbitrary) \\
$\beta$ & allometric constant & -0.75 & - \\
$\gamma$ & another allometric constant & -0.4 & - \\
$\epsilon$ & assimilation efficiency & 0.4 & - \\
$g_{0}$ & maximum predation rate of organism in smallest size class & 1.4 & $\mathrm{~d}^{-1}$ \\
$\delta_{0}$ & mortality rate of predators in smallest size class & 0.068 & $\mathrm{~d}^{-1}$ \\
$r_{0}$ & maximum growth rate of prey in smallest size class & 1.4 & $\mathrm{~d}^{-1}$ \\
$P_{s}$ & full-saturation prey density & 2 & $\mathrm{mmol} \mathrm{m}^{-3}$ \\
$\lambda$ & mortality rate (size-independent component) & 0.016 & $\mathrm{~d}^{-1}$ \\
$K$ & carrying capacity & 2.43 & $\mathrm{mmol} \mathrm{m}^{-3}$ \\
\hline \hline
\end{tabular}



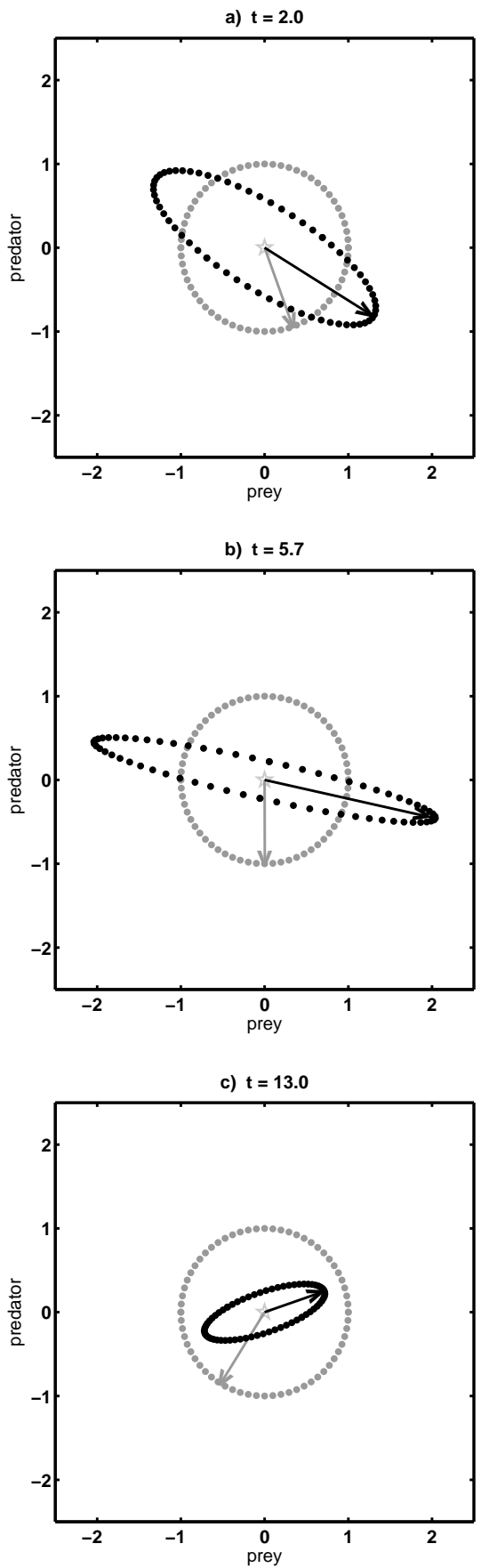

Figure 1: Transient amplification of perturbations in the predator-prey model (54-55) at $t=2.0$ (showing growth of perturbations), $t=5.7$ (maximum of the amplification envelope), and $t=13$ (as perturbations decay). Initial conditions (with unit norm) are represented by gray dots; the response is shown by black dots. Parameters from Table 1, Equilibrium A. 


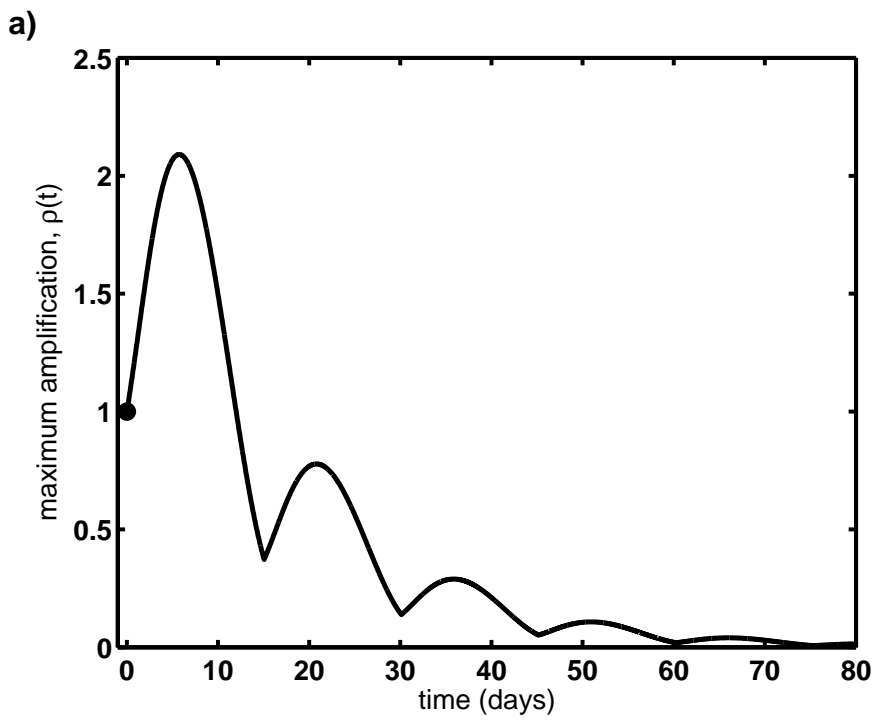

b)

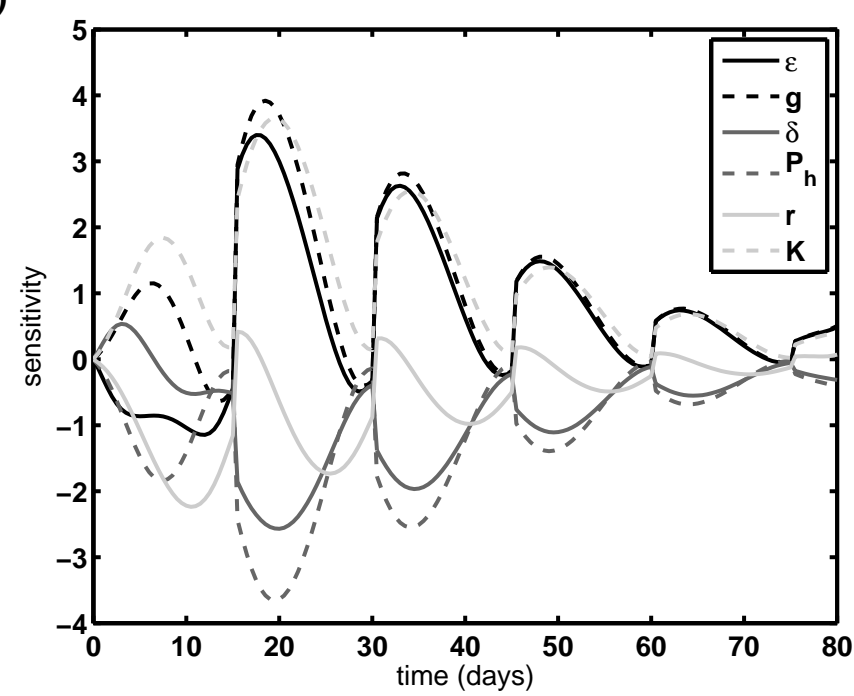

Figure 2: (a) The amplification envelope $\rho(t)$ for Equilibrium A of the predator-prey model $(54-55)$; the equilibrium is reactive $\left(\nu_{0}=0.24\right)$. Maximum growth occurs at $t=5.7$. (b) The sensitivity of the amplification envelope to the log of each parameter, as a function of time. 
a) reactivity

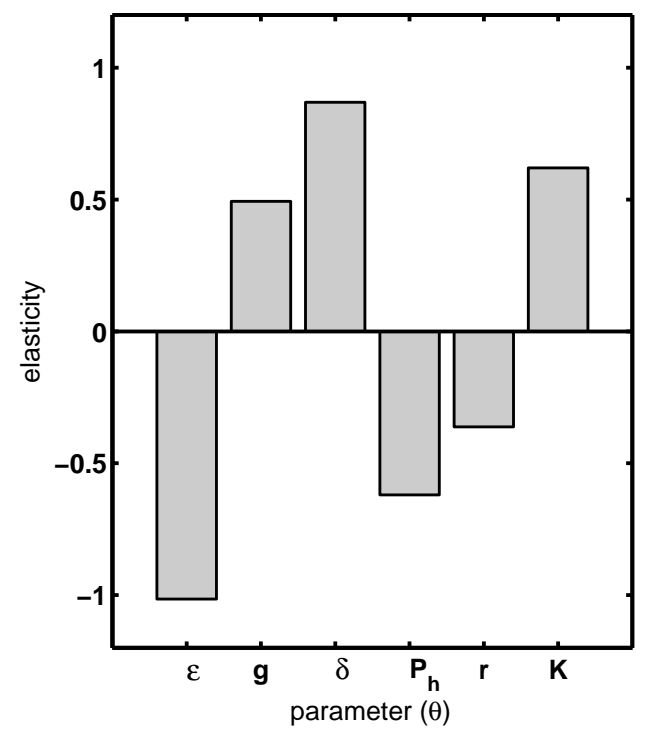

b) amplification envelope, $t=5.7$

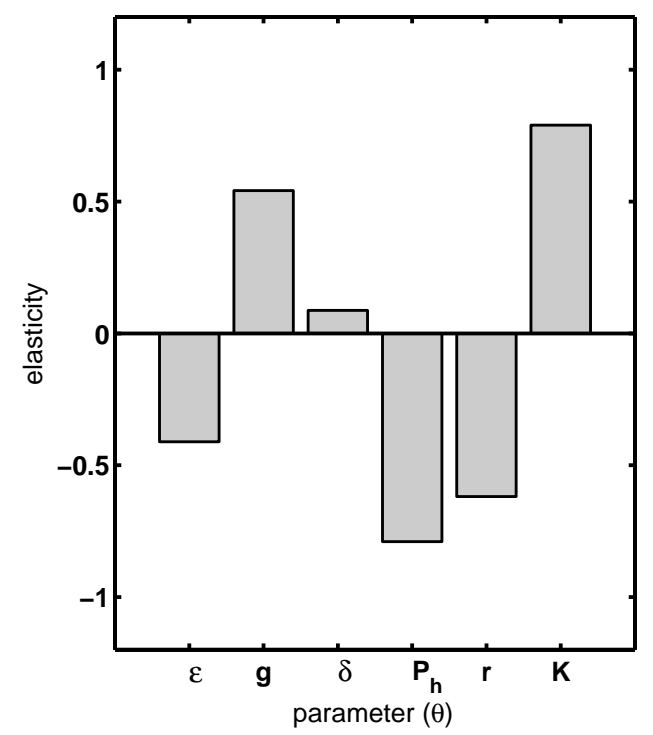

Figure 3: Perturbation analysis of transient dynamics for Equilibrium A of the predatorprey model (54-55). (a) The elasticity of reactivity. (b) The elasticity of the amplification envelope at the time of maximum amplification. 


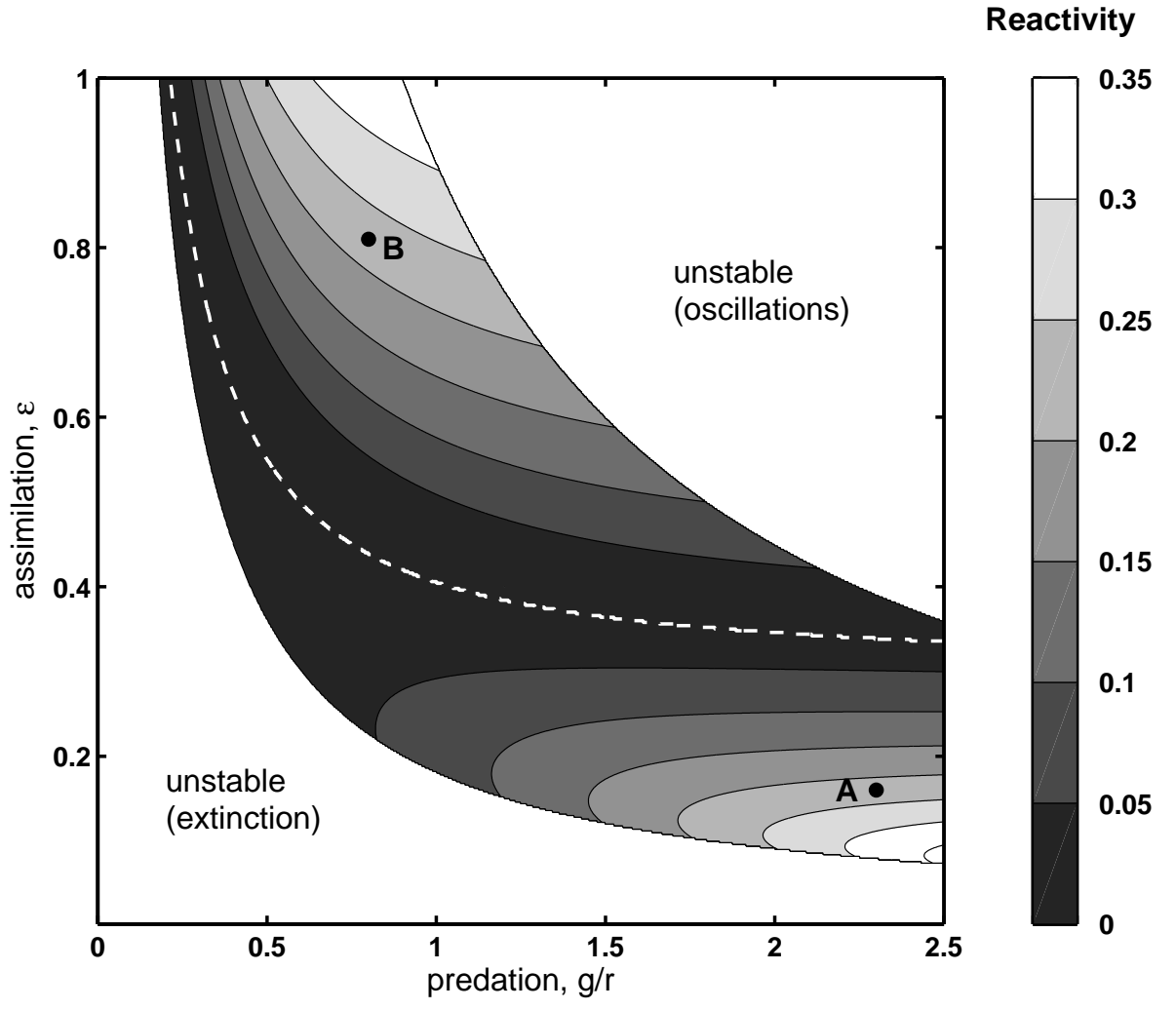

Figure 4: Reactivity $\nu_{0}$ of the predator-prey model (54-55) in parameter space. Shades of gray give the value of $\nu_{0}$ in region where the coexistence equilibrium is stable. The white dashed line indicates a ratio of $\hat{Z} / \hat{P}=3$ (above the curve, ratio is higher). Equilibria A and B (see Table 1) have identical reactivities, but different mechanisms are responsible for transient growth. 

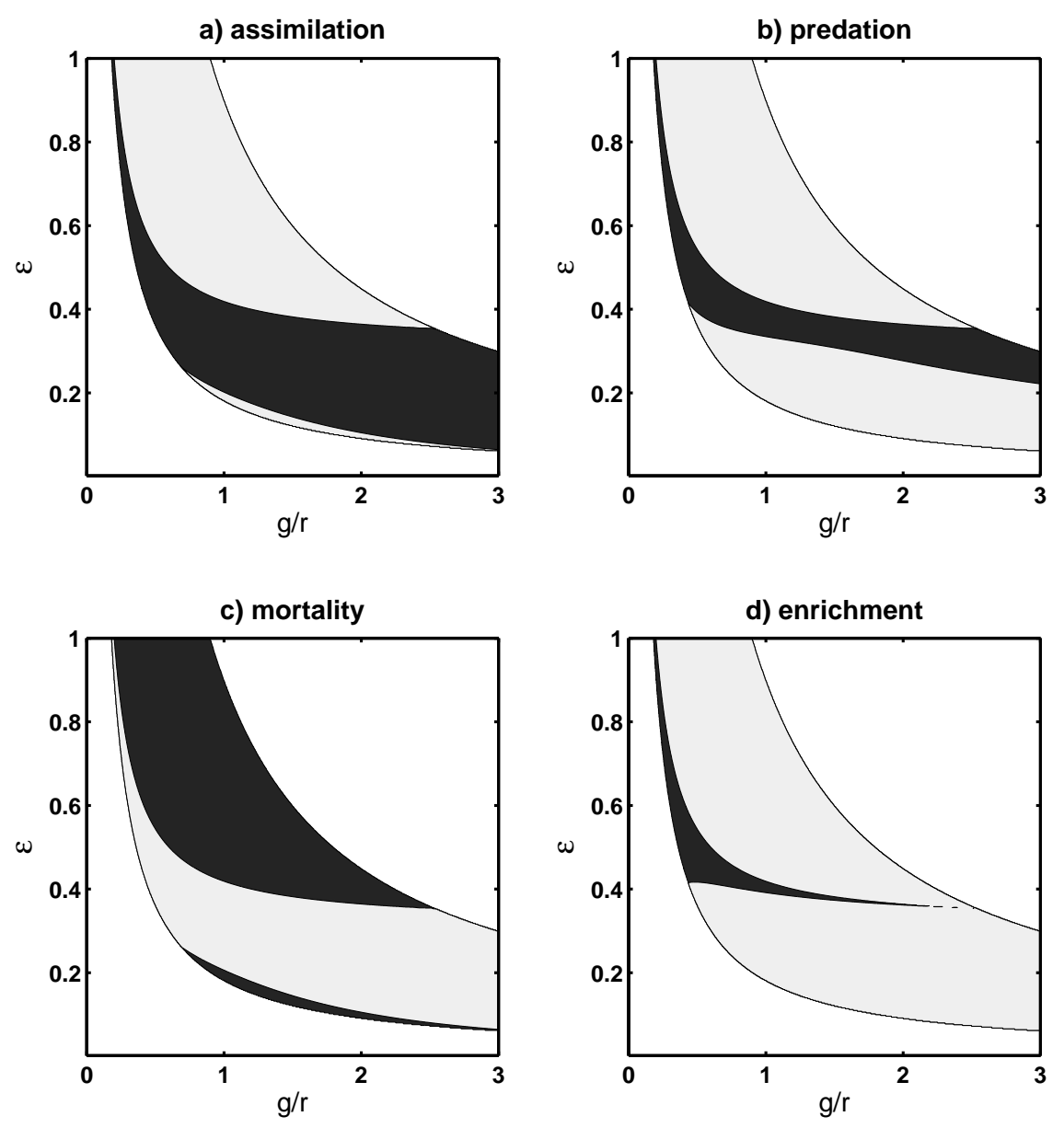

Figure 5: The sign of the sensitivity of reactivity of the predator-prey model (54-55) in the region of parameter space where the coexistence equilibrium is stable. Dark areas indicate negative sensitivity to the non-dimensional parameters, light gray areas indicate positive sensitivity. Sensitivity of $\nu_{0}$ to (a) assimilation $(\epsilon)$, (b) predation $(g / r),(\mathrm{c})$ mortality $(d / r)$, and (d) enrichment $\left(K / P_{h}\right)$. 
a) Equilibrium $A$

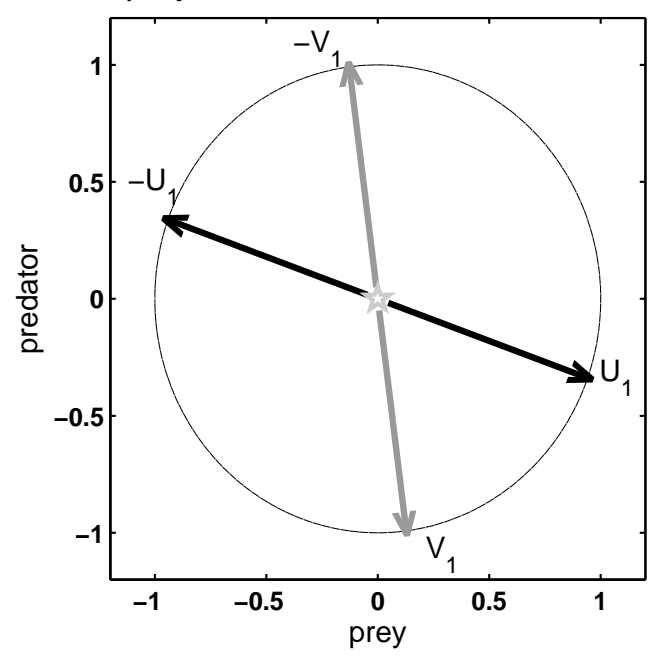

b) Equilibrium $B$

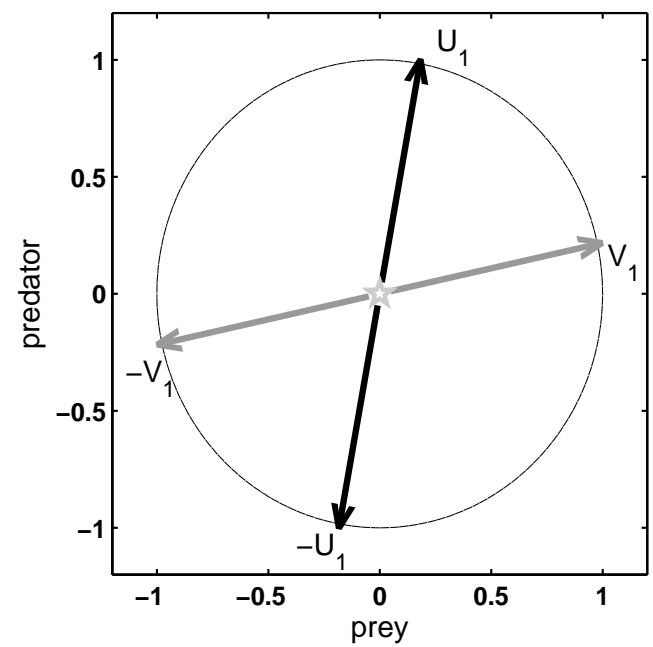

Figure 6: Optimal perturbations and the corresponding responses for equilibria A and B of the predator-prey model $(54-55)$ at $t=4$. Optimal perturbation is shown in gray; the response at $t=4$ is shown in black. 


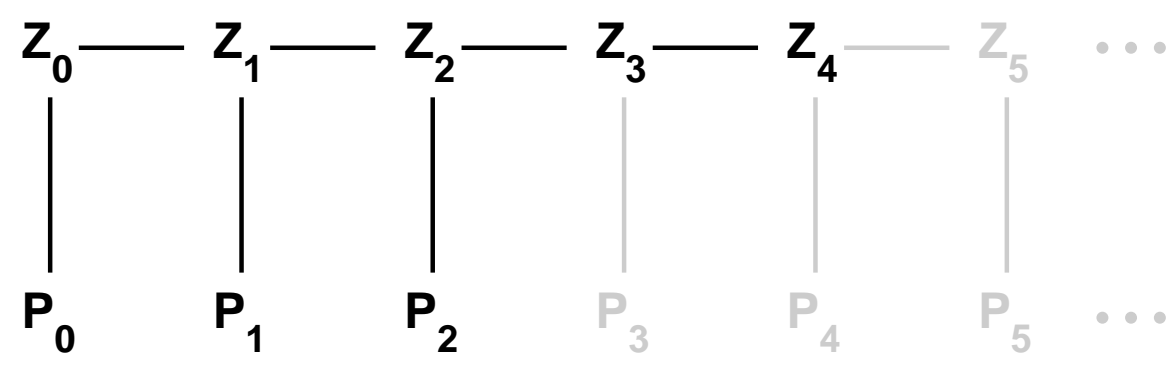

Figure 7: Size-structured multiple food chains model, inspired by Armstrong (1994). Zooplankton in a given size class can feed on a single class of phytoplankton, as well as on the next smaller zooplankton class. For the parameters of Table 2, the system supports 3 phytoplankton classes $\left(P_{i}\right.$ for $\left.i \leq 2\right)$ and 5 zooplankton classes $\left(Z_{i}\right.$ for $\left.i \leq 4\right)$. Larger organisms (shown in gray) become extinct.

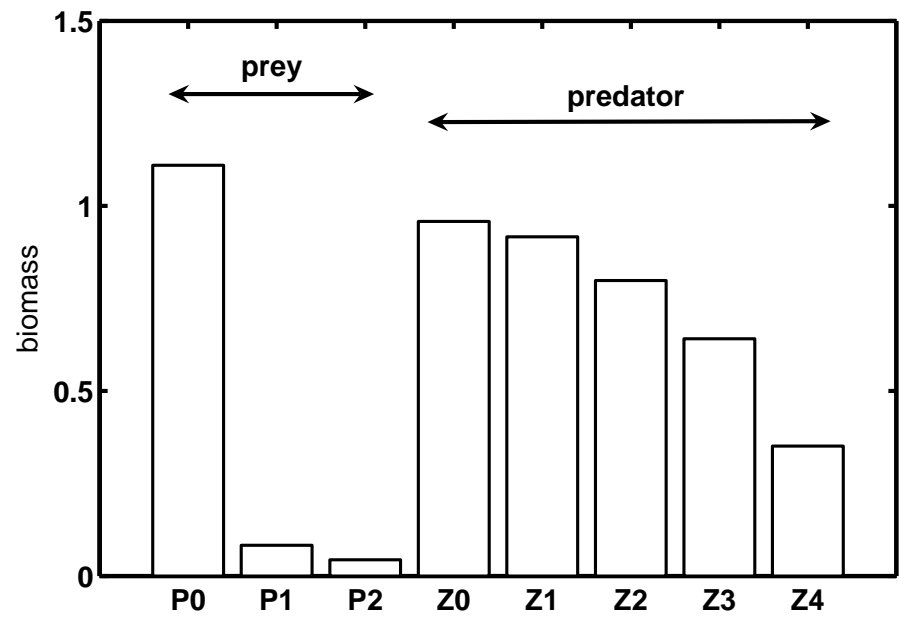

Figure 8: Equilibrium densities in the size-structured multiple food chains model (61-62), for the parameters given in Table 2 . 


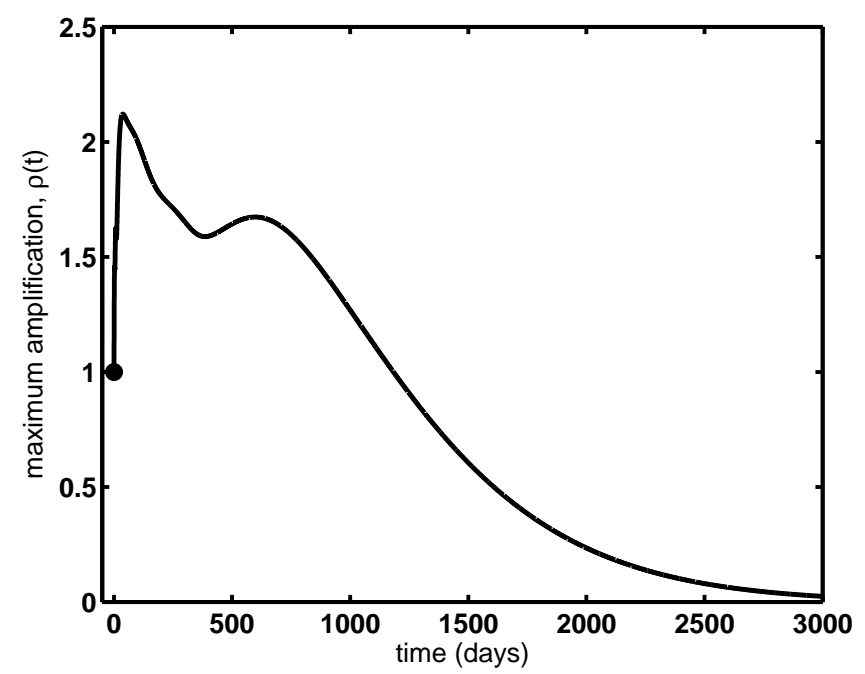

Figure 9: The amplification envelope for the size-structured multiple food chains model (61-62); the equilibrium is reactive $\left(\nu_{0}=0.29\right)$. Maximum transient growth occurs at $t=37$.

a) reactivity

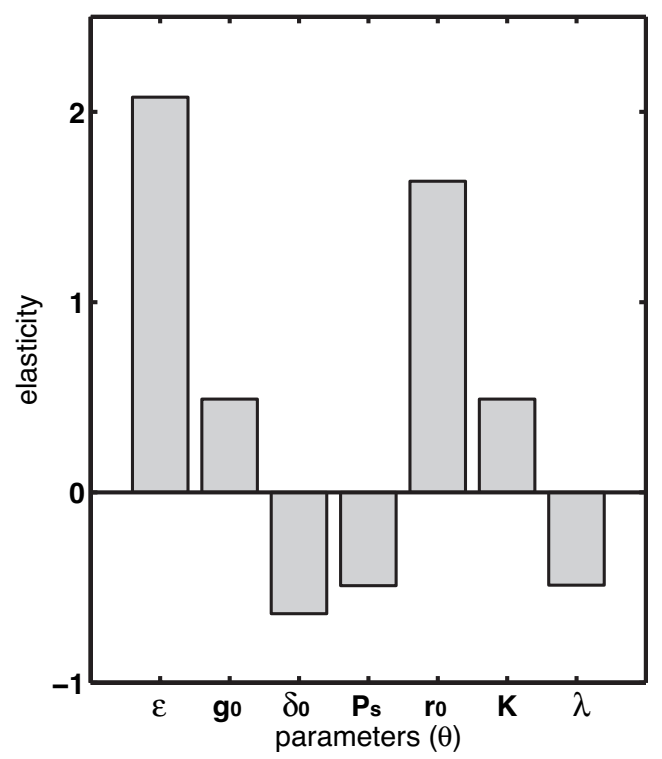

b) amplification envelope, $t=37$

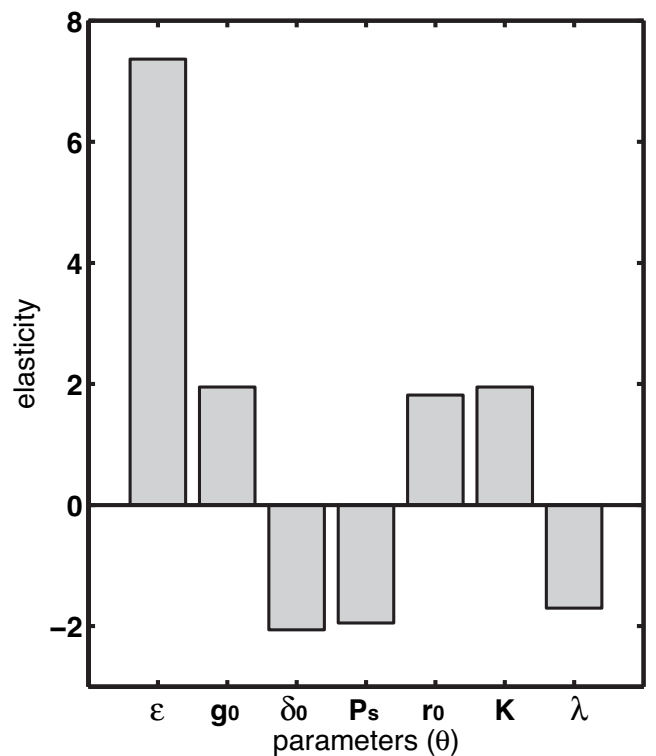

Figure 10: Perturbation analysis of transient dynamics for the size-structured multiple food chains model (61-62). (a) The elasticity of reactivity. (b) The elasticity of the amplification envelope at the time $(t=37)$ of maximum amplification. 